\title{
Supporting Informaiton
}

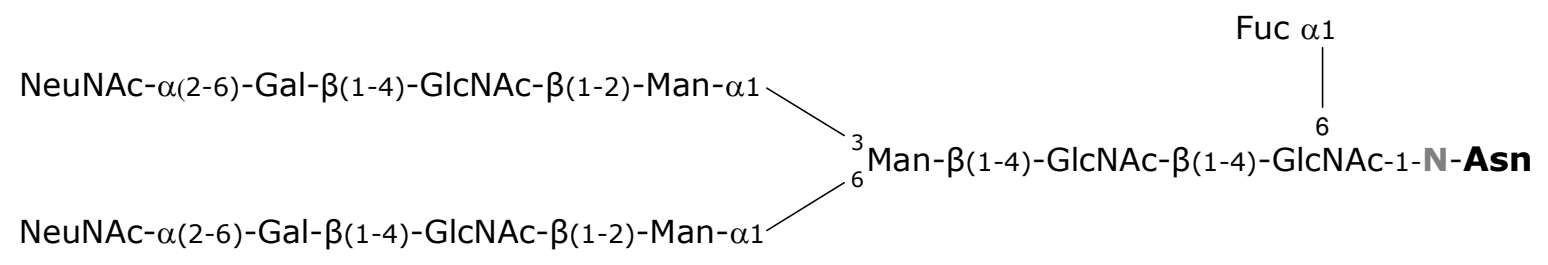

Figure S1. Carbohydrate chain connected to residue Asn60g of thrombin. Asn: asparagine, GlcNAc: N-acetylglucosamine, Fuc: fucose; Man: mannose, Gal: galactose; NeuNAc: Nacetylneuramic acid. 


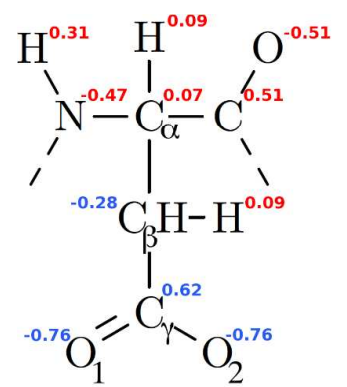

(a)

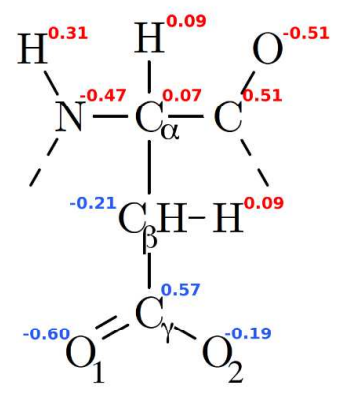

(d)

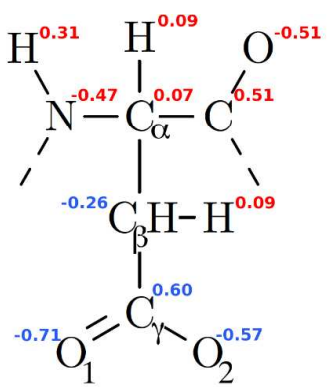

(b)

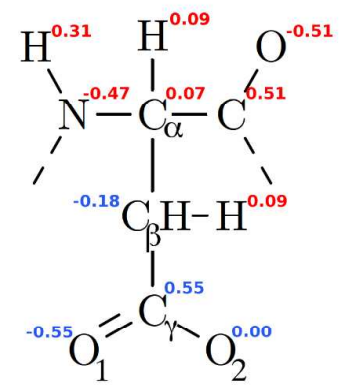

(e)

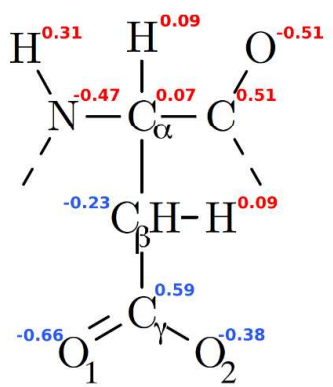

(c)

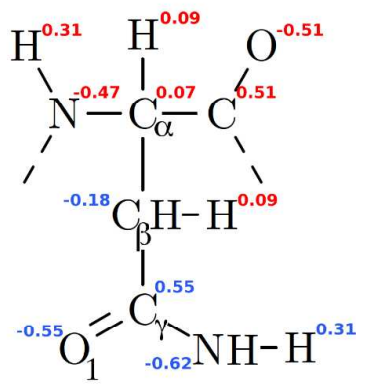

(f)

Figure S2. Schematic protocol employed for the alchemical morphing of Asp102 into Asn102. (a e) Charge morphing part of the protocol. Partial atomic charges are reported near the atoms; in red are the charges that were kept fixed during the morphing as in the CHARMM27 force field they are the same for both Asp and Asn. Charges in blue, instead, were modified according to the linear interpolation $q_{i}(\xi)=(1-\xi) q_{i}^{(\mathrm{Asp})}+\xi q_{i}^{(\mathrm{Asn})}$. The parameter $\xi$ assumed the values (a) 0 , (b) 0.25 , (c) 0.5 , (d) 0.75 , and (e) 1 . For $\xi=0$, the Asp residue charges are assigned to all the Asp102 atoms. For $\xi=0$, an artificially mutated Asp102 residue is created in which the atoms have the charges of Asn (the second carboxylic oxygen collects the global 0 charge of the $-\mathrm{NH}_{2}$ moiety). (f) Final step of the alchemical mutation, where one of the two carboxylic atoms of Asp is changed instantaneously into the $-\mathrm{NH}_{2}$ moiety, to which the correct charges are assigned. All the charges are given in units of the absolute value of the electron charge. 